\title{
An Optimal Control Load Demand Sharing Strategy for Multi-Feeders in Islanded Microgrid
}

\author{
Muhammad Zahid Khan ${ }^{1}$, Muhammad Mansoor Khan², Xu Xiangming ${ }^{3}$, Umar Khalid ${ }^{4}$, Muhammad Ahmed Usman \\ Rasool $^{5}$ \\ ${ }^{1,2,4,5}$ School of Electronic, Information and Electrical, Engineering, Shanghai Jiao Tong University (SJTU), Shanghai, China \\ ${ }^{3}$ State Grid Zhenjiang Power Supply Company, Jiangsu, China
}

\begin{abstract}
For the operation of autonomous microgrid (MG), an essential task is to meet the load demand sharing using multiple distributed generation (DG) units. The conventional droop control methods and its numerous variations have been developed in the literature in order to realize proportional power sharing amongst such multiple DG units. However, the conventional droop control strategies are subjected to power sharing error because of non-trivial feeder impedances of medium-voltage MGs. Further, complex MGs configurations (mesh or looped networks) usually make to reactive power sharing and system voltage regulation more challenging. This paper presents an optimal control strategy in order to perform the proportional power sharing and voltage regulation for multiple feeders in islanded AC MGs. The case study simulation for optimizing the power sharing and voltage regulations in proposed control strategy has been verified through using MATLAB/Simulink systems.
\end{abstract} MG

Keywords-Optimal control; power sharing; voltage regulation;

\section{INTRODUCTION}

The application of distributed power generation such as wind turbine, photovoltaics' and fuel cell has been experienced a fast development in the past decades [1] [2]. DG units as compare to conventional centralized power generation, provides more clean and renewable power close to consumer's end [3][4]. Therefore, it can ease the stress of numerous traditional transmission and distribution framework[5]. Power electronics converters are interfaced between DG units and the grid, are the vital elements of the MGs [6] , and perform the flexibility of islanded or grid connected operation.

On the other side, high infiltration of power electronics based DG units presents couple of issues, such as voltage deviations, frequency and power flow variations [7]. In order to sort out these aforementioned problems, the idea of MG has been emerged, which is based on the control of multiple DG units. As compare to a solitary DG, MG can accomplish predominant power management within its distribution network [3]. The MG can operate either in grid-connected mode or islanding mode. In grid connected mode, the MG is connected with the main grid at the point of common coupling (PCC) and according to dispatched references every DG unit provides proper active and reactive power. The most used control strategies are reported in [8] for grid connected inverters.
In the operation of islanded MG mode, the load demand should be appropriately shared by DG units according to their respective ratings and availability of power from either their respective prime movers or energy sources [3]. Communication based power sharing control strategies include master/slave control, concentrated control, and distributed control[8], while control strategies without communication are usually based on the droop concept, which can be classified into four main categories: 1) conventional and variants of the droop control[9]; 2) construct and compensate based strategies[10]; 3) the hybrid droop/signal injection strategy; 4) In [11] virtual framework structure-based method is developed.

Traditional frequency and voltage magnitude droop control approaches are adopted for interfacing inverters in a decentralized mode to attain power sharing and voltage regulation [12]. However, a little while back is observed that conventional droop control strategy in low voltage MG has led to have few power control stability issues, as the DG feeders have largely resistive (high $\mathrm{R} / \mathrm{X}$ ration) behavior [7]. It can also be observed that active power at the steady state is usually proportionally shared among DG units, while the reactive power sharing deteriorates due to mismatched of DG units output and feeder impedances [7]. The impedances of transmission line be asymmetrical due to distinctive separations amongst DG units while the design of LCL filters are depends on varying system conditions and design considerations which leads to dissimilar DG unit's output filters impedances [13] [7]. In addition, the presence of local loads and the complex network MG configuration usually further increase load sharing performance.

To resolve the power control problems, few enhanced droop control strategies [11] and [14] have been reported in previous literature. In [15], an accurate power sharing control approach has been reported to restore the load point voltage with the decreased voltage deviation. Author proposed an enhanced reactive sharing strategy in[5]. Aforementioned, these two strategies are, however, attained at cost of inverter terminal voltage deviation. Furthermore, the droop strategies based on virtual impedances methods are seen as a promising strategy to handle power sharing issue. The virtual frequencyvoltage frame and virtual power idea were reported in [14] [14]and [11], that enhance the stable operation of the MG system. However, these strategies cannot subdue the reactive power sharing errors in the meantime. In addition, appropriate power sharing among inverter and electric machine is subject 
to challenging in these strategies, when small synchronous generators are included into the MG. Although the author addressed the power sharing issue in[16], but the respective steady state voltage distortion deteriorates the overall power quality of MG. Author proposed an "Q-V dot droop” strategy in [13], but it is noticed that reactive power sharing enhancement is not evident when the local loads are incorporated. Author in [17] used additional PCC voltage measurement in order to mitigate the error of reactive power sharing. In [18], an enhanced virtual impedances control method has been reported for a DG unit, that is able to compensate for the unequal feeder impedances. Although, the power sharing can be enhanced by virtual impedance based droop control strategies but voltage droop and virtual impedance deteriorates the inverter terminal voltage quality [19].

In order to reduce the tradeoff among reactive power sharing and bus voltage deviations in multi feeders a recent control strategy is developed in [20] where a Kalman filterbased state estimator used which required high bandwidth date rate. In addition, feeders can be located at considerable distance from each other, therefore it increases complexity and reduce the reliability and flexibility of MG operation. Therefore, this paper proposed an optimal control load demand sharing strategy for multi-feeders which is directly based on load estimation and optimal regulator as shown in Fig. 3. The salient contribution of this work can summarized as follows:1) The load is estimated at respective feeders which reduces the bandwidth data requirements; 2) The proposed optimal control strategy achieved task of proportional power sharing and system voltage regulation for multiple buses simultaneously.

The rest of this paper is organized as follows. In Section II, the operation of MG is discussed. The operating principal of a proposed control approach is given in section III. The simulations results are presented in section IV and finally section V concludes the paper.

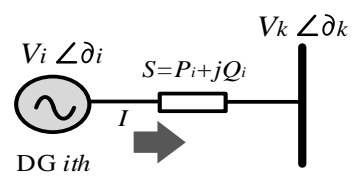

Fig. 1. An $i^{\text {th }}$ Inverter Connected with $k^{\text {th }}$ AC Bus.
MGs are consisting on considerable number of DG units and connected load as shown in Fig. 2. Every DG unit is connected to the MG with an interfaced inverter where DG inverters connected to the PCC via their corresponding feeders. The statues of main grid and MG are controller by the MG central controller. Depending on operations requirements, the main grid can be connected or disconnected from the MG by switching the state of static transfer switch STS at the common bus coupling point. In the grid-connected operation mode, the active and reactive reference usually are allocated by central controller and in order to track the power the conventional droop control strategy can be used. PI regulation for the voltage magnitude control used to mitigate the steady stated reactive power tracking errors. So, during grid connected mode the power sharing is not concern. When micro grid is operating in islanded mode, the load demand of MG should be properly shared by DG units. In this mode of operation, the DG units can operate using conventional power frequency droop control strategies as

$$
\begin{aligned}
& \omega_{i}=\omega_{i}^{*}-D_{P i} \cdot P_{i} \\
& V_{i}=V_{i}^{*}-D_{Q_{i}} \cdot Q_{i}
\end{aligned}
$$

Where, $V_{i}^{*}, \omega_{i}, D_{P}$ and $D_{\mathrm{Q}}$ are the nominal voltage magnitude, nominal frequency, real and reactive power slops, respectably for $i^{\text {th }} \mathrm{DG}$ unit.

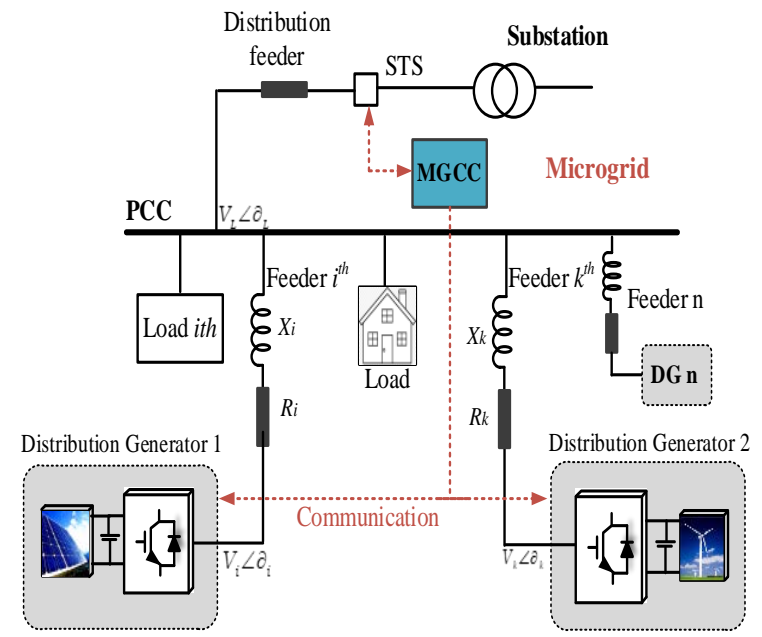

Fig. 2. Illustration of the Microgrid Configuration.Operation of MG.

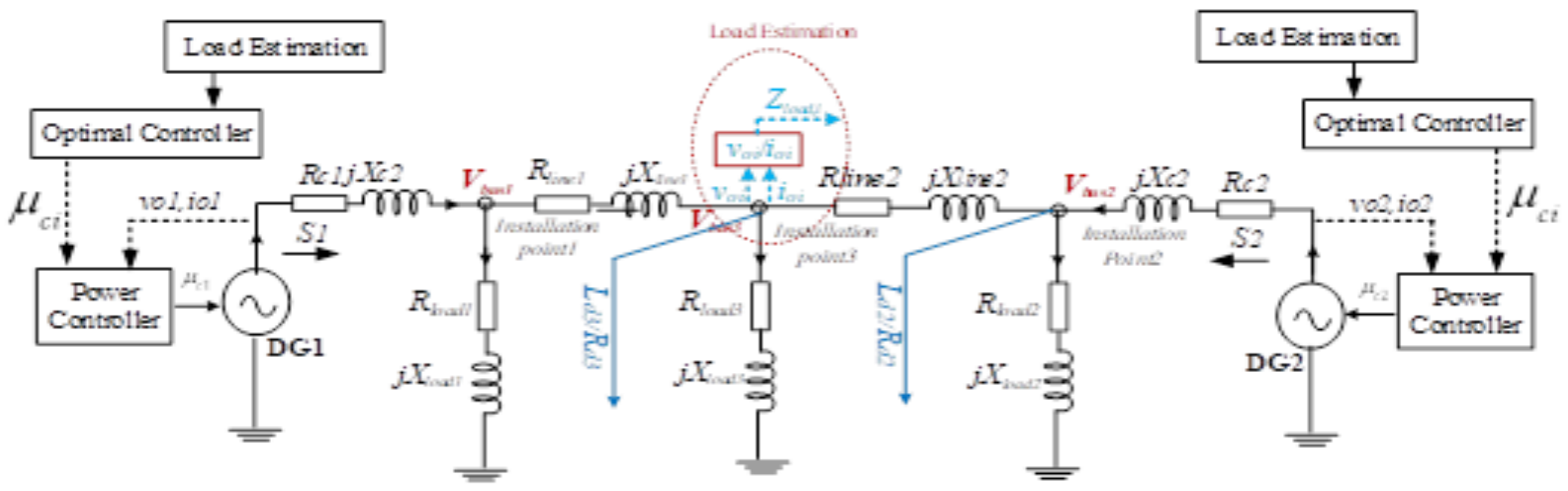

Fig. 3. Block Diagram of Proposed Optimal Control Strategy. 


\section{Proposed Optimal Control StRATEgy}

A radial type feeder is used in proposed optimal control strategy as illustrated in Fig. 2. All three buses $\mathrm{V}_{\text {bus1 }}, \mathrm{V}_{\text {bus2 }}$, and $\mathrm{V}_{\text {bus3 }}$ are fed through two DG units DG1 and DG2 interfaced using three phase, three wire power electronics inverters connecting through feeder impedances with three linear loads $\mathrm{R}_{\text {load1}}, \mathrm{R}_{\text {load2 }}$ and $\mathrm{R}_{\text {load3 }}$. This proposed strategy is composed on load estimation and optimal steady state estimator regulator. Load estimation strategy used in order to estimate specific feeder's impedances which have advantages that it reduces the data bandwidth requirements. Based on these load estimation values $\mathrm{Z}_{\text {load,i, }}$ the optimal regulators are responsible to compute the optimal control command which is a cost function and send two optimal control commands $\mathrm{u}_{\mathrm{c} 1}$ and $\mathrm{u}_{\mathrm{c} 2}$ to power controllers in order to realize proportional power sharing and voltage restoration. Ld2/Rd2 and Ld3/Rd3 are disturbance load which are exerted to examine the effectiveness of this proposed strategy for both inductive and resistive MGs. Different cases of disturbance load addition are further discussed in detail in section 4 .

\section{A. Mathematical Model}

In order to explain operation of a MG, a simplified circuit in Fig. 1, is illustrated where two $i^{\text {th }}$ and $k^{\text {th }}$ DG units are parallel connected. The complex power drawn towards the $k^{\text {th }}$ ac bus can be expressed as.

$S_{i k}=P_{i k}+j Q_{i k}$

Where, active power $\mathrm{P}_{\mathrm{i}}$ and reactive power $\mathrm{Q}_{\mathrm{i}}$ are introduced at every existing node by DG converters. If power inverters are supposed to be an ideal controllable voltage source which is connected to line impedances via mains, then the movement of real and reactive powers in transmission line impedances can be expressed as.

$P_{i}=\frac{V_{i}}{R_{i}^{2}+x_{i}^{2}} \cdot\left[R_{i} V_{i}-R_{i} V_{k} \cos \partial_{i k}+X_{i} V_{k} \sin \partial_{i k}\right]$

$Q_{i}=\frac{V_{i}}{R_{i}^{2}+x_{i}^{2}} \cdot\left[-R_{i} V_{k} \sin \partial_{i k}+X_{i} V_{i}-X_{i} V_{k} \cos \partial_{i k}\right]$

Where, $\mathrm{i}=1,2$ represents the two branches in circuit. $\mathrm{V}_{\mathrm{i}}$ is magnitude of inverter output and $\mathrm{V}_{\mathrm{k}}$ represents the PCC bus voltage, while $\mathrm{P}_{\mathrm{i}}$ and $\mathrm{Q}_{\mathrm{i}}$ are the active and reactive powers flowing from $\mathrm{i}^{\text {th }}$ inverter terminal to $\mathrm{k}^{\text {th }}$ common bus voltage, illustrates the difference amongst the power angle phase of the output impedance.

In higher voltage $\mathrm{HV}$ and medium MV network the inductive elements are typically higher then resistive as shown in table 1 [11], however, HV and MV networks have inductive behavior, therefore we can neglect the resistive part. As power angle $\partial$ is small in such type of lines so we can assume that and the possible power flow in network can be written as

$P_{i, R_{x}=0} \approx \frac{V_{i} V_{k}}{x_{i}}\left[\sin \partial_{i k}\right]$
$Q_{i, R_{x}=0} \approx \frac{V_{i}^{2} V_{k}-V_{i} V_{k} \cos \partial_{i k}}{x_{i}}$

Where,

$\partial_{i}-\partial_{k} \propto P_{i}$

$V_{i}-V_{k} \propto Q_{i}$

According to expression (8) and (9), it is obvious that the real power $\mathrm{P}_{\mathrm{i}}$ drawn towards $k^{\text {th }}$ node predominately depends on power angle while reactive power $\mathrm{Q}_{\mathrm{i}}$ injected by each DG inverter mostly controlled by voltage difference $V_{i}-V_{k}$ of ith and kth ac bus.

\section{B. Load Estimation and Optimal Regulator Principal}

Proposed optimal control strategy used to estimate the load at specified feeder follows variable frequency local voltage based park transformation as expressed in (10) and elaborated in Fig. 4(a). This strategy, firstly sensed the voltage $V_{\text {bus,i }}$ and current $\mathrm{I}_{\text {bus,i }}$ at local node of $i^{\text {th }}$ feeder, later both voltage and current signals are converted into abc-dq0, where rotating frame is aligned 90 degrees behind A axis. dq0 values converted from real-imaginary inputs to a complex valued output signal, where load impedances $\mathrm{Z}_{\mathrm{ri}-\mathrm{c}}$, $\mathrm{I}$ is achieved by ratio of $\mathrm{V}_{\mathrm{i}} \angle \partial_{\mathrm{i}}$ and $\mathrm{i}_{\mathrm{i}} \angle \partial_{\mathrm{i}}$ which is also a complex valued signal as expressed in (11). Since, optimized cost function impedances which is expressed in (13), input signals should be real-imaginary valued signal, so a complex to real-imaginary block is used as shown in Fig. 4(a), and elaborated in (12), which converts the complex load impedances signals to realimaginary valued impedances signals. Aforementioned, these impedances are estimated at respective feeders which reduce the bandwidth data requirements. Later these impedances signals are sent to the proposed optimal controller achieved task of proportional power sharing and voltage restoration for multiple buses simultaneously.

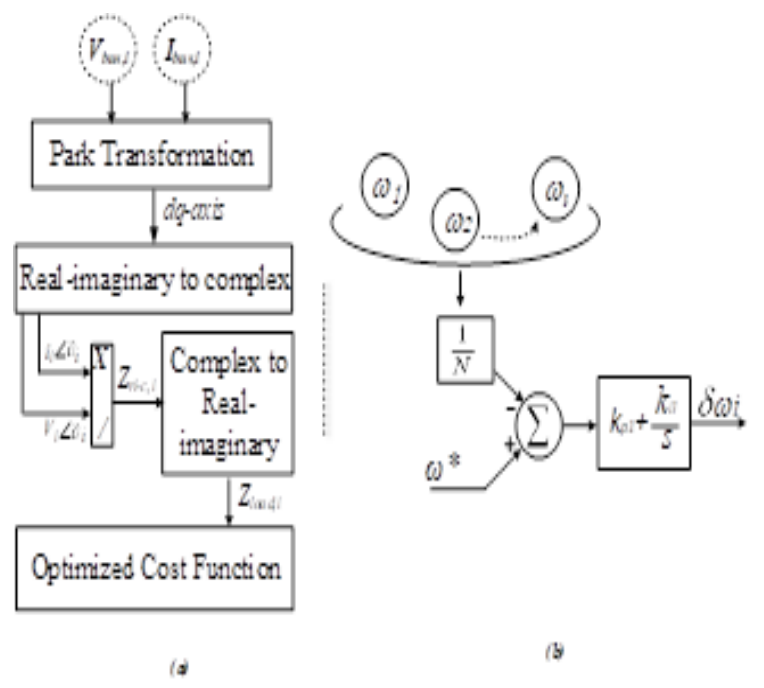

Fig. 4. (a) Load Estimation of $\mathrm{i}^{\text {th }}$ Feeder (b) Frequency Regulation. 


$$
\begin{aligned}
& \left.U_{s}=\left(u_{d}+j \cdot u_{q}\right)=\left(u_{\alpha}+j \cdot u_{\beta}\right) \cdot e^{-j\left(\omega t-\frac{\pi}{2}\right)}\right) \\
& \left.\left[\begin{array}{l}
u_{d} \\
u_{q} \\
u_{0}
\end{array}\right]=\left[\begin{array}{lcc}
\sin (\omega t) & -\cos (\omega t) & 0 \\
\cos (\omega t) & \sin (\omega t) & 0 \\
0 & 0 & 1
\end{array}\right]\left[\begin{array}{l}
u_{a} \\
u_{b} \\
u_{c}
\end{array}\right]\right\} \\
& \left.V_{\text {mag }}=\sqrt{\left(V_{\text {Real }}\right)^{2}-\left(V_{\text {Imag }}\right)^{2}} ; \partial_{i}=\tan ^{-1} V_{\text {Real }} / V_{\text {Imag }}\right) \\
& \left.i_{\text {mag }}=\sqrt{\left(i_{\text {Real }}\right)^{2}-\left(i_{\text {Imag }}\right)^{2}} ; \partial_{i}=\tan ^{-1} i_{\text {Real }} / i_{\text {Imag }}\right\} \\
& V_{\text {Real }}=V_{i} \cos \angle \partial_{i} ; \quad V_{\text {Imag }}=V_{i} \sin \angle \partial_{i} \\
& \left.i_{\text {Real }}=i_{i} \cos \angle \partial_{i} ; \quad i_{\operatorname{Im} a g}=i_{i} \sin \angle \partial_{i}\right\}
\end{aligned}
$$

Once the load impedances founded, the optimal regulator is a following critical step. The proposed optimal regulators are an optimized cost function which is presented in order to compute control commands according to the estimated network impedances. Different type of optimization techniques is being used such as linear, quadratic and higher order optimization strategies in order to minimize or maximize cost function of the system. Linear optimization has wide area of application and it is easy regarding solvability but the limitation of linear optimization is that it works only with the variables that are linear as well as problem formulation is freaky. Higher order cost functions are convenient but solution is inconvenient. In this paper, quadratic optimization based cost function is used which is easy regarding solvability, problem formulation and solution is also convenient. Some cost functions have constrained reactive powers which can be supposed to equal i.e $\mathrm{Q}_{1}=\mathrm{Q}_{2}$ in order to vary the tradeoff among real power and inverter terminal voltages, but usually the reactive power requirement is not so stringent, so in this case, this paper used constrains real powers which are supposed to be equal $\mathrm{P}_{1}=\mathrm{P}_{2}$ and the tradeoff among bus voltage and reactive powers is found through minimization. Moreover, the desired control commands are acquired by computing the optimization cost function that minimize the reactive power sharing error $\Delta Q_{i}$ and voltage error $\Delta V_{i}$ at specific bus, which can be expressed as

$$
\min J=\left(\sum_{j=1}^{n_{b}}\left(\omega_{b j}\left(V_{b u s_{j}}-V_{j r e f}\right)\right)^{2}\right)+\left(\omega_{Q}\left(Q_{i}-Q_{i+1}\right)\right)^{2}
$$$$
\text { constrainsts } P_{i}-P_{i+1}=0
$$

In the cost function $\omega_{\mathrm{bj}}$ and $\omega_{\mathrm{Q}}$ are the weights for network voltages at specific bus and reactive power error, respectively. $V_{\text {jref }}(j=1,2,3)$ are set at nominal voltage $300 \mathrm{~V}$, while $n_{b}=3$ is the number of buses that has been chosen. Nevertheless, the limitation with is proposed strategy is that, it requires the accurate grid impedances of every load node which aggravates the computationally complexity with increase in feeder numbers. By considering that limitations, in future the whole grid network impedance can approximated into one load node which will reduce the computationally burden over system.

However, moving forward, after computing every optimal controller sends desired control commands to their respective power controllers and operates with the control commands until upcoming sampling update. Frequencally, the optimal regulator obtains new estimated impedances due to measurements feedback and accordingly revises its original control plan. Then, the voltage control commands are send to compensate for voltage and reactive power sharing deviations.

\section{Power Flow Control}

Proposed control strategy is illustrated in Fig. 5. The output frequency and voltages of Inverter Bridge connected with dc power source are adjusted by power, voltage and current controllers. Every individual DG unit is formulated in its $\mathrm{d}$-q frame where they depend on their both individual angular frequency and angle. Every inverter interfaced with DG units are transferred to the d-q frame by using following transformation equations as

$$
\left[\begin{array}{l}
f_{D} \\
f_{Q}
\end{array}\right]=\left[\begin{array}{cc}
\cos (\partial i) & -\sin (\partial i) \\
\sin (\partial i) & \cos (\partial i)
\end{array}\right]\left[\begin{array}{l}
f_{d} \\
f_{q}
\end{array}\right]
$$

The power controller block for proposed strategy is illustrated in Fig. 5, adopts P- $\omega$ droop as illustrated in (15) and provides the angle of $i^{\text {th }}$ DG units which can be express as

$$
\begin{aligned}
& \omega_{i}=\omega_{i}^{*}-m_{P i} P_{i} \\
& \partial_{i}=\int\left(\omega_{i}-\delta \omega_{i}\right) d t
\end{aligned}
$$

Where $\omega^{*}{ }_{i} \neg$ and $m_{\mathrm{Pi}}$ are the reference frequency and the P$\omega$ droop coefficient, respectively. Noticeably, the voltage references $\mathrm{u}_{\mathrm{ci}}$ as shown in Fig. 5, is used instead of Q-V droop, which can be calculated by optimization based cost function as discussed aforementioned in section B. However, the average real power is acquired by instantaneous power passing low-pass filters as expressed below

$$
P_{i}=\frac{\omega_{c}}{s+\omega_{c}} p_{i}
$$

Where, $\omega_{c}$ is cutoff frequents of low pass filters Instantaneous active power pi can be represented in d-q frame as

$$
p_{i}=V_{o d i} \cdot i_{o d i}+V_{o q i} \cdot i_{o q i}
$$

Here, $\mathrm{v}_{\text {odqi }}$ and $\mathrm{i}_{\text {odqi }}$ are inverter terminal voltage and current, respectively on $\mathrm{d}-\mathrm{q}$ frame.

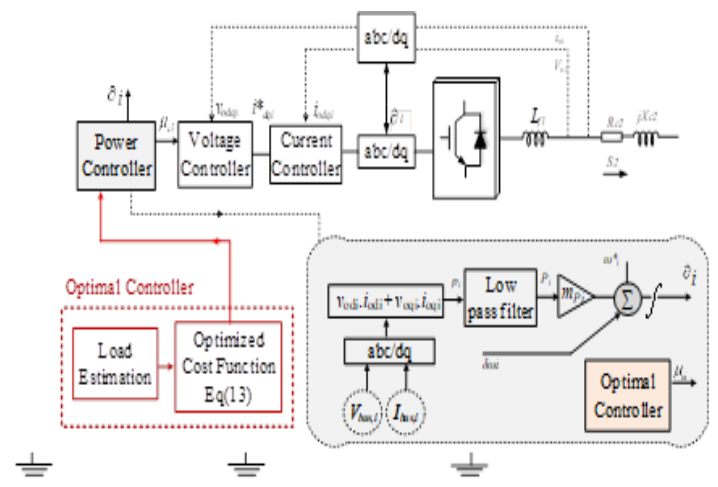

Fig. 5. Proposed Optimal Control Strategy. 


\section{Frequncy Regulation}

The secondary controllers for MGs are based upon frequency restoration. Since, the real power highly influences the frequency of generator-dominated grids. This feature is an edge, since frequency is controllable variable that gives the information regarding to generation or consumption balance of the grid. The frequency regulation strategy which is implemented in order to restore the frequency of system is illustrated in Fig. 4(b) which regulates the frequency deviations of $i^{\text {th }}$ DGs units to its nominal value. Frequency restoration strategy can be expressed by $\omega^{*}$ and $\omega_{\text {avg }}$ are the nominal reference frequency and measure system frequency that is being sensed by each node of DGs unit's interface inverters in the neighborhood of the node i being considered. Frequency correction is send to frequency reference of the $i^{\text {th }}$ inverters node, while $\mathrm{K}_{\mathrm{pf}}$ and $\mathrm{K}_{\mathrm{i}}$ are the proportional and integral gains, respectively, for controllers.

$$
\begin{aligned}
& \overline{\omega_{\text {avg }}}=\frac{\sum_{k=1}^{N} \omega_{k}}{N} \\
& \omega_{i}=\left(\omega^{*}-\overline{\omega_{\text {avg }}}\right) \\
& \delta \omega_{i}=k_{p f} \omega_{i}+k_{i f} \int \omega_{i} d t
\end{aligned}
$$

\section{E. Mechanism of Reactive Power Sharing}

The output of the optimizer is set of voltage phasors and its implementations require the information of voltage phasers of all inverters participating the MG. A direct reconstruction of such information needs very fast and reliable communication and computation infrastructure. However, in presented approach the phases have been modified through frequency/real power droop control and only voltage magnitudes are updated in accordance with the optimizer output. This combination will render accelerates active power sharing which is also considered as constraint in cost function. Consequently, the correct phase angle will automatically be adjusted by system.

\section{RESUlt AND DisCUSSION}

In order to verify the effectiveness of proposed control approach, the simulations have been carried out on MATLAB/Simulink for a three phase $50-\mathrm{Hz}$ islanded MG. As illustrated in Fig. 6 the simulated MG is composed on two DG1 and DG2 unites connected in parallel with three linear loads via feeder impedances. The circuit and control parameters are shown in Fig. 6 and Table 1, respectively. Simulation verifications are composed on two cases. The case 1 and case 2 investigates the effectiveness of proposed control strategy on different disturbance locations in inductive and resistive MG, respectively.

TABLE I. TYPICAL LINE IMPEDANCES

\begin{tabular}{|l|l|l|l|}
\hline Type of Lines & $\mathbf{R}(\mathbf{\Omega} / \mathbf{k m})$ & $\mathbf{X}(\mathbf{\Omega} / \mathbf{k m})$ & $\mathbf{R} / \mathbf{X}$ \\
\hline Low voltage line & 0.642 & 0.083 & 7.7 \\
\hline Medium voltage line & 0.161 & 0.190 & 0.85 \\
\hline High voltage line & 0.06 & 0.191 & 0.31 \\
\hline
\end{tabular}

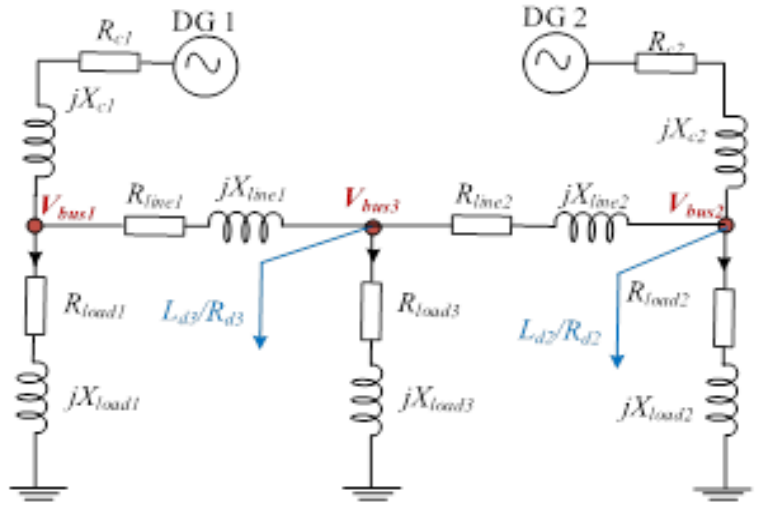

Fig. 6. Proposed Circuit Configuration.

\section{A. Case 1: Optimal Control Strategy for Inductive MG}

In this section, results obtained from proposed strategy and without proposed strategy for inductive are discussed. The key parameters and configuration for inductive MG are given

\begin{tabular}{|c|c|c|}
\hline Parameters & Symbol & Value \\
\hline Frequency & $f s$ & $50 \mathrm{~Hz}$ \\
\hline Inverter Rating & $V A$ & $10 \mathrm{kVA}$ \\
\hline Filter 1 impedances & $\mathrm{R}_{\mathrm{c} 1}+\mathrm{j} \mathrm{X}_{\mathrm{c} 1}$ & $0.25+j 0.785$ \\
\hline Filter 2 impedances & $\mathrm{R}_{\mathrm{c} 2}+\mathrm{j} \mathrm{X}_{\mathrm{c} 2}$ & $0.25+j 0.785$ \\
\hline Line 1 impedances & $\mathrm{R}_{\text {line1 }}+\mathrm{j} \mathrm{X}_{\text {line1 }}$ & $0.2+j 0.628$ \\
\hline Line 2 impedances & $\mathrm{R}_{\text {line2 } 2}+\mathrm{j} \mathrm{X}_{\text {line2 }}$ & $0.2+j 0.628$ \\
\hline Load 1 impedances & $\mathrm{R}_{\text {load1 } 1}+\mathrm{j} \mathrm{X}_{\text {load1 }}$ & $20+j 3.140$ \\
\hline Load 2 impedances & $\mathrm{R}_{\text {load } 2}+\mathrm{j} \mathrm{X}_{\text {load2 }}$ & $20.4+j 3.15$ \\
\hline Load 3 impedances & $\mathrm{R}_{\text {load3 }}+\mathrm{j} \mathrm{X}_{\text {load3 }}$ & $20+j 3.140$ \\
\hline Disturbance load at Bus 2 & $\mathrm{~L}_{\mathrm{d} 2} / \mathrm{R}_{\mathrm{d} 2}$ & $10 \mathrm{mH} / 19$ \\
\hline Disturbance load at Bus 2, 3 & $\begin{array}{l}\mathrm{L}_{\mathrm{d} 2} / \mathrm{R}_{\mathrm{d} 2} \\
\mathrm{~L}_{\mathrm{d} 3} / \mathrm{R}_{\mathrm{d} 3}\end{array}$ & $7+j 0.314,10+j 0.628$ \\
\hline
\end{tabular}
in table 2 and Fig. 6, respectively

TABLE II. SYSTEM PARAMETERS FOR INDUCTIVE MICROGRID

1) Power Sharing with load variation at bus 2: In this case, the all buses voltage error weight wa-c and reactive power error weight $\omega \mathrm{Q}$ are not changed and set at 1 which does not have any effect on cost function. In order to realize proportional power sharing and verify the optimal control strategy a heavy disturbance load $\mathrm{Ld} 2 / \mathrm{Rd} 2$ with value of 19+j3.140 exerted at bus 2 on 0.2 seconds. Fig. 7 and 8 depicts the performance of voltage regulations and power sharing, respectively, with and without proposed control strategy. In conventional control strategy the bus voltages drop (dotted curves) can be seen in Fig. 7(a-b). since droop controllers decrease voltages in order to track the aggravated reactive power. More than 3 volts' deviation at bus 2 and bus 3 has been compensated in proposed strategy as illustrated in Fig. 7(a-b) and stabled at 297.2 V and 297.56 V, respectively. In addition, active power sharing error $4.3 \mathrm{KW}$ and reactive power sharing error 710VARcan be noticed in Fig. 7(c) and Fig. 8(b). Once the proposed optimal control strategy is activated at 0.2 seconds, it can be observed in Fig. 8(a and c) that active and reactive power sharing error are compensated in to almost zero with a smaller startup divergent behavior. 


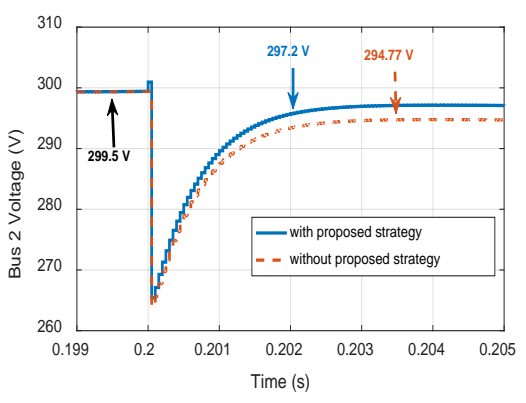

(a)

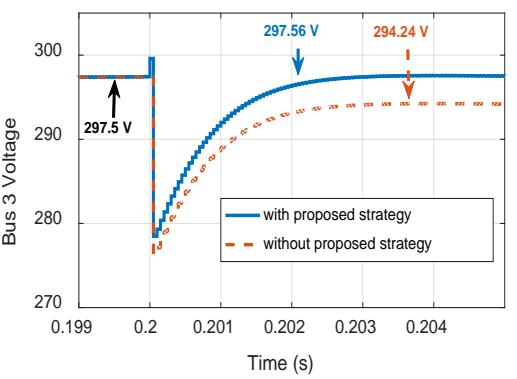

(b)

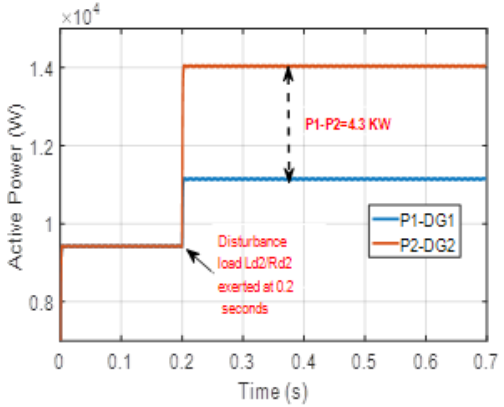

(c)

Fig. 7. Simulations Results under Heavily Load Conditions (a)Voltage Response at Bus 2 (b) Voltage Response at Bus 3 (c) Active Power Sharing without Proposed Strategy.

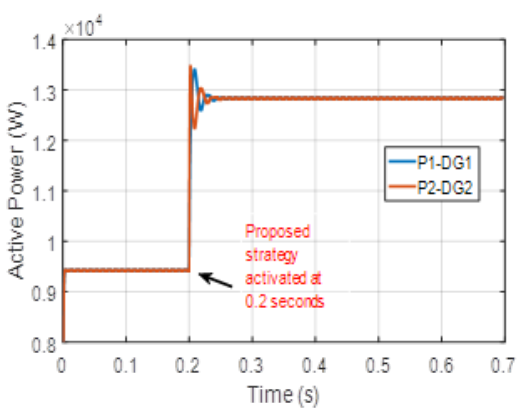

(a)

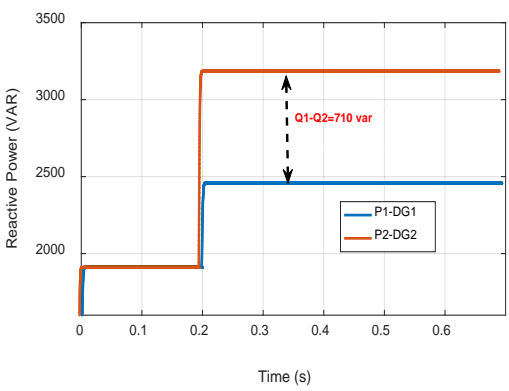

(b)

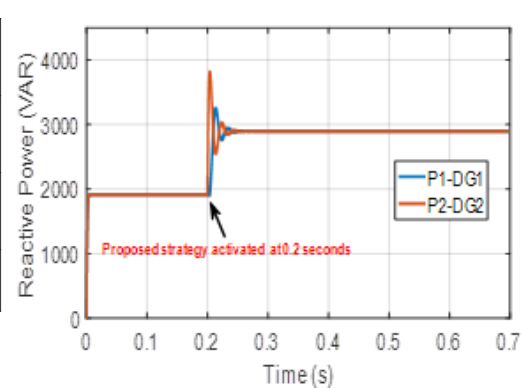

(c)

Fig. 8. (a)Active Power Sharing in Proposed Strategy, Reactive Power Sharing in with and without Proposed Strategy is Illustrated in Figure (b) and (c), respectively.

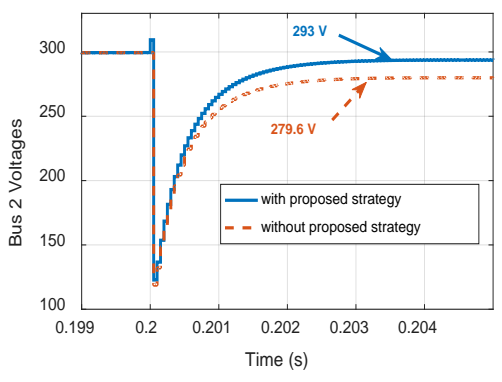

(a)

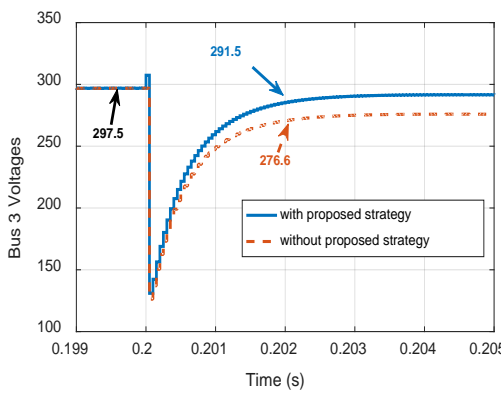

(b)

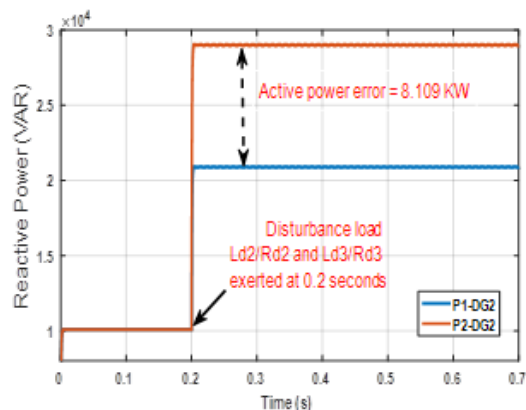

(c)

Fig. 9. Simulations Results under Heavily Load Conditions with (Green Cure) and without (Yellow Dotted Line) Proposed Control Strategy (a)Voltage Response at Bus 2 (b) Voltage Response at Bus 3 (c)Active Power Sharing without Proposed Strategy.

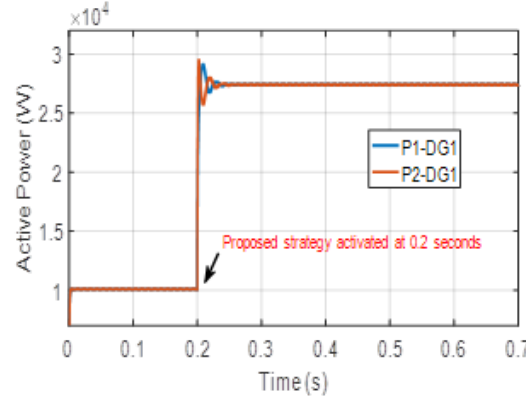

(a)

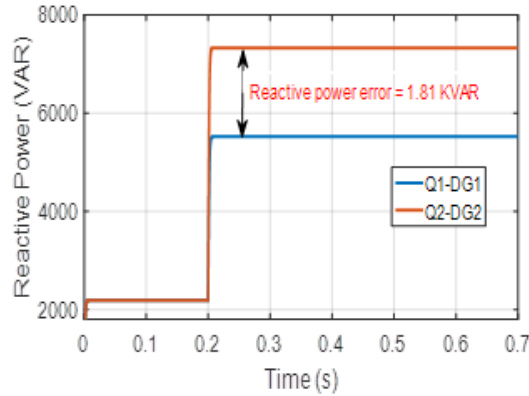

(b)

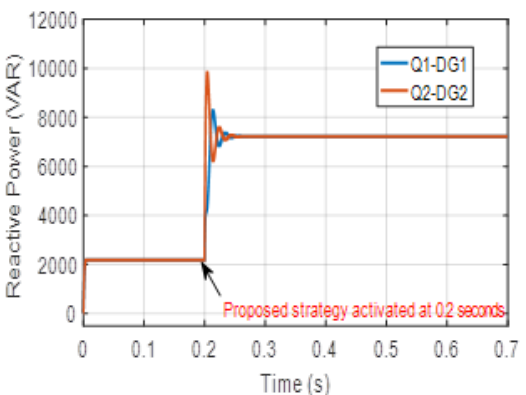

(c)

Fig. 10. Simulation Results with Proposed Strategy for active Power is Shown in Figure (a), while Results Obtained with and without Proposed Strategy for Real and Reactive Power are Illustrated in (b) and (c), respectively. 
2) Power Sharing for different disturbance locations: This section further investigates the effectiveness of system voltage regulations and power sharing behavior to unknown multiple disturbances. In this case, a serious disturbance load $(\operatorname{Ld} 2 / \operatorname{Rd} 2)$ and $(\operatorname{Ld} 3 / R d 3)$ are exerted on same time on 0.2 seconds at bus 2 and 3, respectively, as shown in Fig. 5 which led up to20.4v and 23.4Volts deviations at bus 2 and bus 3, respectively. Proposed strategy is activated at 0.2 seconds, which reduces deviations of $13.4 \mathrm{~V}$ at bus 2 and stabled voltage curve at $293 \mathrm{~V}$ as illustrated in Fig. 9 (a), while 14.9V deviation has been compensated at bus 3 as shown in Fig. 9(b), and voltage curve is stabled within acceptable +$0.5 \mathrm{~V}$ range. Further, multiple disturbance load at different locations effects power sharing, as active power error $8.01 \mathrm{Kw}$ and reactive power error 1.08KVAR is noticed in Fig. 10(c) and 10(b). When the proposed control strategy is activated at 0.2 seconds power sharing error is compensated to almost zero as shown in Fig. 9 and 10. Optimal Control Strategy for Resistive MG.

TABLE III. SYSTEM PARAMETERS FOR RESISTIVE MICROGRID

\begin{tabular}{|l|l|l|}
\hline Parameters & Symbol & Value \\
\hline Filter 1 impedances & Rc1+jXc1 & $1.5+\mathrm{j} 0.314$ \\
\hline Filter 2 impedances & Rc2+jXc2 & $1.5+\mathrm{j} 0.314$ \\
\hline Line 1 impedances & Rline1+jXline1 & $0.75+\mathrm{j} 0.157$ \\
\hline Line 2 impedances & Rline2+jXline2 & $0.75+\mathrm{j} 0.157$ \\
\hline Load 1 impedances & Rload1+jXload1 & $30+\mathrm{j} 3.140$ \\
\hline Load 2 impedances & Rload2+jXload2 & $30.4+\mathrm{j} 3.15$ \\
\hline Load 3 impedances & Rload3+jXload3 & $35+\mathrm{j} 3.140$ \\
\hline Disturbance load at Bus 2 & Ld4/Rd4 & $1 \mathrm{mH} / 20$ \\
\hline
\end{tabular}

To further investigates the effectiveness of proposed optimal control strategy, the results are obtained for resistive MG as shown in Fig. 11, 12 and 13. System configuration and parameters for resistive MG are illustrated in Fig. 6 and table 3 respectively. In this case II, the objective of optimal control strategy is to realize proportional power sharing and hold bus 2 voltages at its nominal voltage $V_{\text {ref }}$ valued $300 \mathrm{~V}$, in the presence of load disturbance Ld4/Rd4.

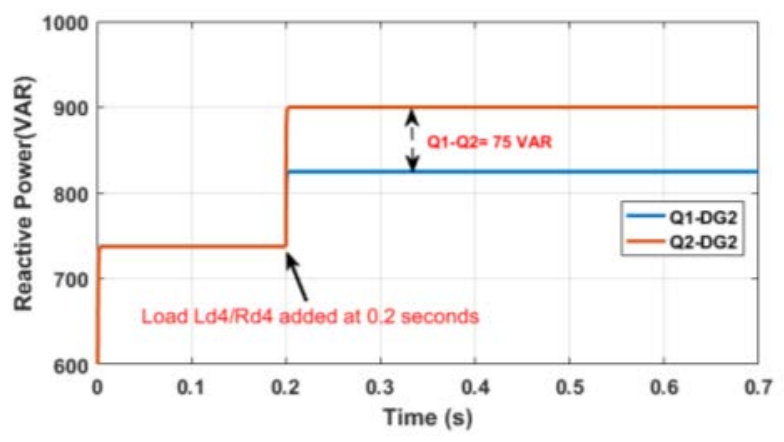

Fig. 11. Reactive Power Sharing without Proposed Control Strategy.

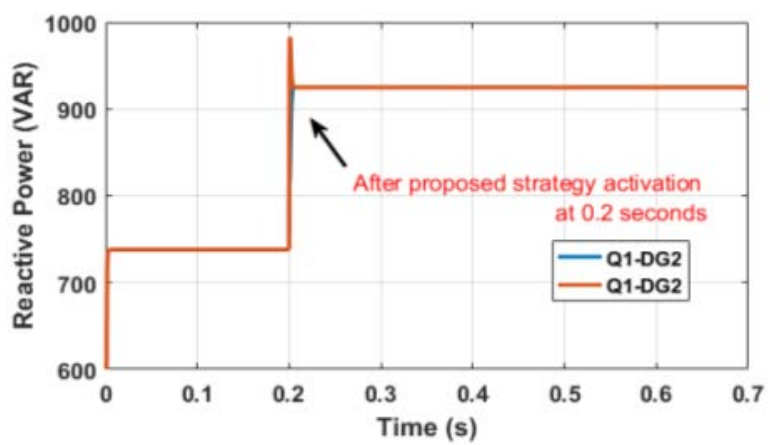

Fig. 12. Reactive Power Sharing with Proposed Control Strategy.

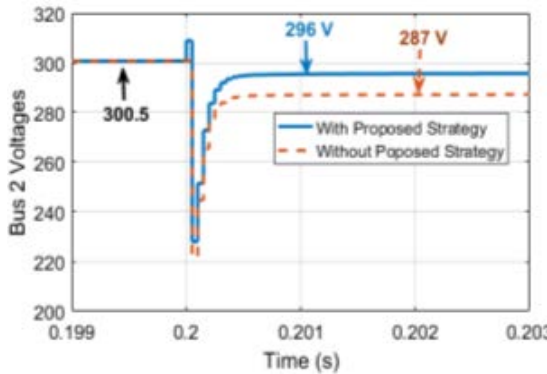

(a)

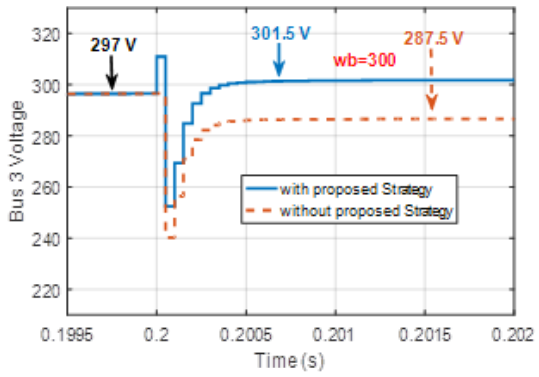

(d)

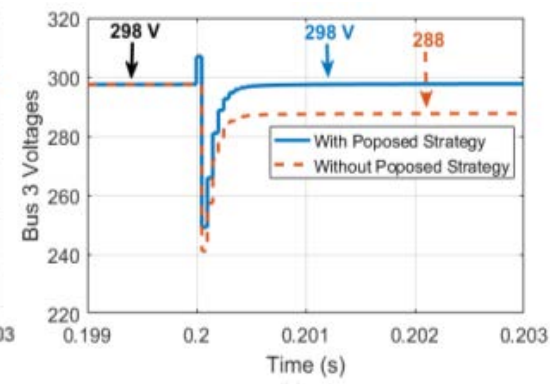

(b)

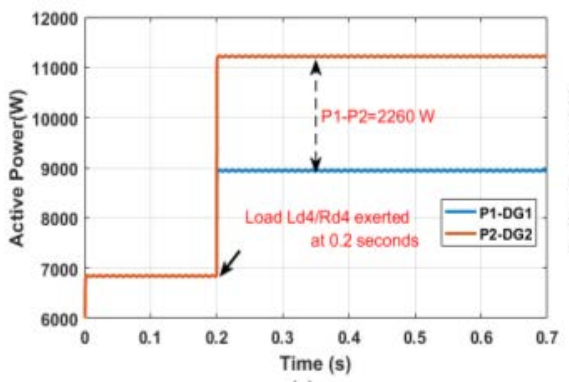

(e)

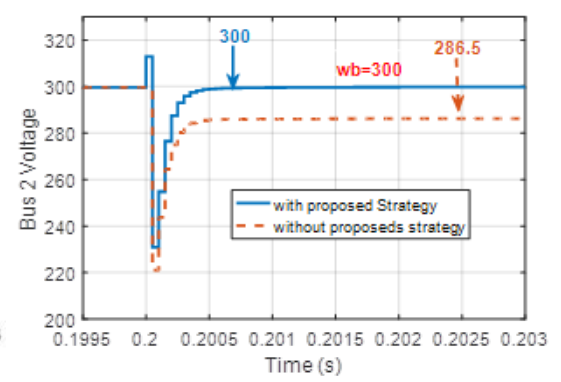

(c)

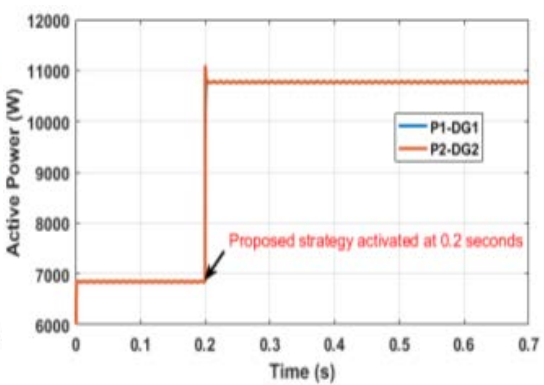

(f)

Fig. 13. Bus 2 and 3 Voltages with and without Proposed Strategy Illustrated in Figure (a) and (b), Respectively, Keeping Weight $\Omega_{b}=1$, While at $\Omega_{b}=300$ is Illustrated in Figure (c) and (d). Figure (e) and (f) Illustrates active Power Sharing without and with Proposed Control Strategy. 
3) Power Sharing and Bus 2 Voltage Control: To validate the optimal control strategy, a disturbance load Ld4/Rd4 with valued $1 \mathrm{mH} / 20 \mathrm{ohm}$ is exerted at bus 2 on 0.2 seconds. Voltage deviation of valued $13 \mathrm{~V}$ and $12 \mathrm{~V}$ is observed in conventional control strategy at bus 2 and 3 . This voltage deviation has been compensated with help proposed control strategy, which stabled bus 2 voltage at $296 \mathrm{~V}$ and bus 3 voltage at $298 \mathrm{~V}$, while keeping weight values $w b=1$ and $\mathrm{wc}=1$ as illustrated in Fig. 13(a- b). Still $4 \mathrm{~V}$ volts deviation occurred at Bus 2 in proposed control strategy as shown in Fig. 13(a). To hold bus 2 voltage at its nominal value Vref in presence of disturbance load, the voltage error weight $\mathrm{Wb}$ is set 300 while all other buses and reactive power weights are set at 1 . The results obtained for Bus 2 after changing of its weight, are shown in Fig. 13(c-d) where it stabled to its nominal value $300 \mathrm{~V}$ voltage. Further, active power error 2260W and reactive power error $75 \mathrm{VAr}$ has been observed in conventional control strategy as illustrated in Fig. 11 and 13(e), respectively. This power sharing error has been compensated to zero in proposed control strategy as depicted in Fig. 12 and Fig. 13(f), respectively.

The strategy adopted for frequency regulation is illustrated in Fig. 4(b). Frequency deviation is eliminated at 0.2 seconds as shown in below Fig. 14, which is within acceptable range $\pm 0.5 \mathrm{~Hz}$.

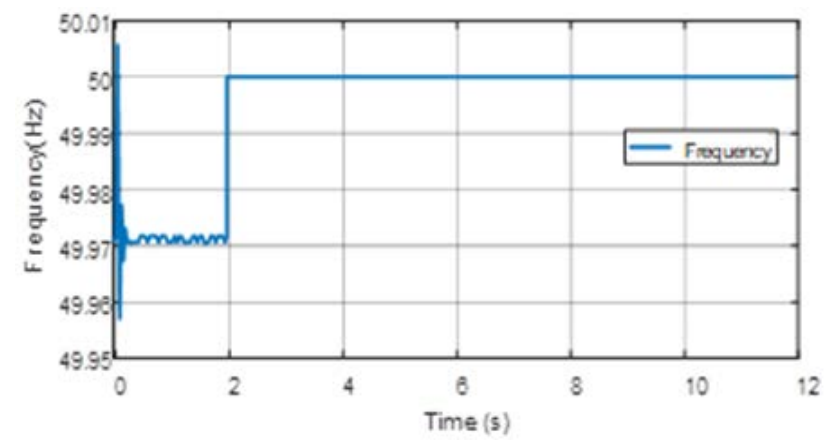

Fig. 14. Frequency Regulation.

\section{CONCLUSION}

In this paper an optimal control strategy was proposed which performs the twofold objectives in order to realize proportional power sharing and system voltage regulation for multiple feeders in islanded AC MGs. The strategy firstly, estimates the load impedances of specified buses by using slow communication channel. Secondly, an optimal controller based optimized cost function with immunity to parameters perturbations has been developed which sends control command to inner loop in order to realize proportional power sharing and voltage control for the specified bus. Finlay, the effectiveness of proposed optimal control strategy was investigated under load parameters uncertainties in both inductive and resistive MGs. The obtained simulation results show that the proposed optimal control strategy is not sensitive to MG's configurations and able to realize proportional power sharing and controls the specified multiple feeder's voltages in ac islanded MG which, thus enhances the reliability and flexibility of islanded MG.

\section{REFERENCES}

[1] S. Member, J. A. Mueller, S. Member, J. W. Kimball, and S. Member, "An Accurate Small-Signal Model of Inverter- Dominated Islanded Microgrids Using dq Reference Frame,” vol. 2, no. 4, pp. 1070-1080, 2014.

[2] R. H. Lasseter and P. Paigi, "Microgrid: A Conceptual Solution," no. June, pp. 4285-4290, 2004.

[3] K. Hashmi et al., “A Virtual Micro-Islanding-Based Control Paradigm for Renewable Microgrids,” pp. 1-23, 2018.

[4] M. Shahid, Muhammad Umair Mansoor Khan, K. Hashmi, S. Habib, J. Huawei, and H. Tang, “A Control Methodology for Load Sharing System Restoration in Islanded DC Micro Grid with Faulty Communication Links,” Electronics, vol. 7, no. 90, pp. 1-15, 2018.

[5] J. He and Y. W. Li, "An Enhanced Microgrid Load Demand Sharing Strategy,” vol. 27, no. 9, pp. 3984-3995, 2012.

[6] F. Blaabjerg, R. Teodorescu, M. Liserre, and A. V. Timbus, "Overview of control and grid synchronization for distributed power generation systems,” IEEE Trans. Ind. Electron., vol. 53, no. 5, pp. 1398-1409, 2006.

[7] M. Umair and S. Id, “An Improved Control Strategy for Three-Phase Power Inverters in Islanded AC Microgrids,” 2018.

[8] Han et al., "Aalborg Universitet Review of Power Sharing Control Strategies for Islanding Operation of AC Microgrids,” I E E E Trans. Smart Grid, vol. 7, no. 1, pp. 200-215, 2016.

[9] W. Yao, M. Chen, J. Matas, J. M. Guerrero, and Z. M. Qian, “Design and analysis of the droop control method for parallel inverters considering the impact of the complex impedance on the power sharing," IEEE Trans. Ind. Electron., vol. 58, no. 2, pp. 576-588, 2011.

[10] C. K. Sao and P. W. Lehn, "Autonomous Load Sharing of Voltage Source Converters,” IEEE Trans. Power Deliv., vol. 20, no. 2, pp. 10091016, 2005.

[11] K. De Brabandere et al., "A voltage and frequency droop control method for parallel inverters,” Power Electron. Spec. Conf. 2004. PESC 04. 2004 IEEE 35th Annu., vol. 4, no. 4, p. 2501-2507 Vol.4, 2004.

[12] J. M. Guerrero, J. C. Vasquez, J. Matas, L. G. De Vicuña, and M. Castilla, "Hierarchical control of droop-controlled AC and DC microgrids - A general approach toward standardization,” IEEE Trans. Ind. Electron., vol. 58, no. 1, pp. 158-172, 2011.

[13] C. T. Lee, C. C. Chu, and P. T. Cheng, “A new droop control method for the autonomous operation of distributed energy resource interface converters,” IEEE Trans. Power Electron., vol. 28, no. 4, pp. 1980-1993, 2013.

[14] G. Modes, A. Mehrizi-sani, G. S. Member, and R. Iravani, "PotentialFunction Based Control of a Microgrid in,” vol. 25, no. 4, pp. 1883-1891, 2010.

[15] J. He and Y. W. Li, “An accurate reactive power sharing control strategy for DG units in a microgrid,” 8th Int. Conf. Power Electron. - ECCE Asia "Green World with Power Electron. ICPE 2011-ECCE Asia, pp. 551556, 2011.

[16] M. N. Marwali, J. Jung, S. Member, and A. Keyhani, "Control of Distributed Generation Systems — Part II : Load Sharing Control,” vol. 19, no. 6, pp. 1551-1561, 2004.

[17] P. Cheng, C. Chen, T. Lee, and S. Kuo, "A Cooperative Imbalance Compensation Method for Distributed-Generation Interface Converters,” vol. 45, no. 2, pp. 805-815, 2009.

[18] J. M. Guerrero, L. G. De Vicuña, J. Matas, J. Miret, and M. Castilla, "Output impedance design of parallel-connected UPS inverters," IEEE Int. Symp. Ind. Electron., vol. 2, no. 4, pp. 1123-1128, 2004.

[19] D. N. Zmood and D. G. Holmes, "Stationary frame current regulation of PWM inverters with zero steady-state error," IEEE Trans. Power Electron., vol. 18, no. 3, pp. 814-822, 2003.

[20] Y. Wang, S. Member, X. Wang, Z. Chen, and S. Member, "Distributed Optimal Control of Reactive Power and Voltage in Islanded Microgrids,” vol. 53, no. 1, pp. 340-349, 2017. 